\title{
Limited value of type III intestinal metaplasia in predicting risk of gastric carcinoma
}

\author{
K C R B RAMESAR, D S A SANDERS, D HOPWOOD \\ From the Department of Pathology, Ninewells Hospital and Medical School, Dundee, Scotland
}

SUMMARY Endoscopic gastric biopsy specimens taken in 1976 from 174 patients were reviewed. Biopsy specimens from 44 patients showed intestinal metaplasia, and subtyping by mucin histochemistry showed that 16 were of type I, 14 of type II, and 14 of type III. Only two of these 174 patients developed gastric adenocarcinoma over the next 10 to 11 years: one with type II and one with type III intestinal metaplasia. Case notes of a separate group of 68 patients with gastric adenocarcinoma diagnosed in 1985 were reviewed for evidence of intestinal metaplasia in a previous gastric biopsy. Only two patients had previously been biopsied; one of these biopsy specimens showed type II intestinal metaplasia and the other showed no intestinal metaplasia.

These findings suggest that subtyping of intestinal metaplasia in endoscopic gastric biopsy specimens is of only limited value in identifying patients at risk of gastric adenocarcinoma who require long term follow up.

Although the association between intestinal metaplasia and gastric carcinoma has long been recognised, ${ }^{1}$ the prevalence of intestinal metaplasia in benign gastric conditions has precluded its use as an aid to the diagnosis of gastric carcinoma. Recent work has focused on the subtyping of intestinal metaplasia using mucin histochemistry. One of these subtypes, variously called colonic type, ${ }^{2}$ sulphomucin positive, ${ }^{3}$ incomplete type with sulphomucins, ${ }^{4}$ type $2 b,{ }^{5}$ and recently, type III $^{6}$ seems to be strongly associated with concurrent gastric carcinoma. The association of type III intestinal metaplasia with intestinal type but not diffuse type gastric carcinoma, the occurrence of minute gastric carcinomas in areas of incomplete intestinal metaplasia, ${ }^{7}$ and other data ${ }^{5}$ have led to suggestions that type III intestinal metaplasia is a premalignant change rather than a reactive phenomenon. ${ }^{5}$ Thus detection of type III intestinal metaplasia may be of value in the early diagnosis of gastric carcinoma. ${ }^{8}$ More importantly, it has been suggested that it may possibly identify a group of patients with an increased risk of developing subsequent gastric carcinoma. ${ }^{9}$ A prospective study designed to answer these questions is in progress ${ }^{6}$ but the results may not be available for several years. Interim guidelines are therefore required for the management of patients with

Accepted for publication 2 June 1987 type III intestinal metaplasia and to determine policy on its routine subtyping in pathology laboratories.

This study assessed the predictive value of type III intestinal metaplasia for gastric carcinoma with a 10 to 11 year follow up of patients biopsied in 1976. We also determined retrospectively the proportion of patients presenting with gastric carcinoma in a single year (1985) who had shown type III intestinal metaplasia in an earlier gastric biopsy specimen and who might therefore have benefited from "anticipation" of their gastric carcinoma.

\section{Material and methods}

All sections of endoscopic gastric biopsy specimens and partial gastrectomy specimens received by the surgical histopathology service of Ninewells Hospital and Medical School in 1976 were reviewed by two of us (DS and KR). Biopsy specimens were discarded from the study if the biopsy showed malignancy, patient identification was inadequate for follow up, or no tissue remained in paraffin blocks. This resulted in 174 patients entering the study. All of these patients lived in the Dundee and Angus districts, an area with a relatively stable population. Haematoxylin and eosin stained sections were screened, and those showing intestinal metaplasia were recut and stained using the alcian blue (pH 2.5) periodic acid Schiff and high iron diamine/alcian blue (pH 2.5) techniques. ${ }^{10}$ These 
methods stain neutral mucins magenta (PAS), acid mucins blue (alcian blue, pH 2.5), and sulphomucins brown-black (high iron diamine). Patients were entered into one of four categories according to the classification of Filipe $e \mathrm{al}^{6}$ : (i) no intestinal metaplasia; (ii) type I intestinal metaplasia (complete, type 1) showing mature absorptive and goblet cells, the latter secreting sialomucins; (iii) type II intestinal metaplasia (incomplete, type $2 a$ ) showing columnar intermediate cells secreting neutral and acid sialomucins, goblet cells secreting sialomucins or sulphomucins, or both; (iv) type III intestinal metaplasia (incomplete, type 2 b) showing intermediate cells secreting sulphomucins, goblet cells secreting sialomucins or sulphomucins, or both.

Identification data for the 174 study patients was checked against the records of the Ninewells Hospital Cancer Registry for cancer registration up to January 1987. We believe this method of follow up to be particularly accurate in this setting: at the mid point of the study period (1981) Tayside region had one of the lowest net civilian migration rates in Scotland ${ }^{18}$; the region is serviced by only two histopathology laboratories, both of which supply registration data direct to the Ninewells Hospital Cancer Registry. Study patients registered for gastric cancer or metastatic carcinoma "primary unknown" were further investigated via case records.

Case records of patients living in the Dundee area and registered for gastric carcinoma in 1985 were reviewed and details of previous gastric biopsy specimens noted. Of 78 patients registered, 68 sets of records were available for study. Mucin histochemistry was performed on previous gastric biopsy specimens.

Statistical analysis was carried out by the unpaired $t$ test and the $\chi^{2}$ test as appropriate.

\section{Results}

Of the 174 study patients, 44 showed intestinal metaplasia: 16 type I, 14 type II, and 14 type III (table). Patients with intestinal metaplasia tended to be older than those without. This age difference reached significance when those with no intestinal metaplasia (mean age 59.2 years) were compared with those with type I intestinal metaplasia (mean age 66.9 years, $\mathrm{p}=0.01$ ) and those with type III intestinal metaplasia (mean age 65.4 years, $p=0.04$ ) but not those with type II intestinal metaplasia (mean age $64 \cdot 2$ years, $p=0 \cdot 1$ ). The sex ratio was roughly equal in all groups except that those with type III intestinal metaplasia were predominantly male. This difference was not significant $\left(\chi^{2} 6.91, p=0.07\right)$.

A search of the Cancer Registry showed 13 possibly relevant registrations among the 174 study patients, falling into five categories: (i) two patients had had gastric carcinoma diagnosed and treated before the 1976 biopsy; (ii) two patients had subsequently developed gastric lymphoma; (iii) in four patients gastric carcinoma was diagnosed histologically within six months of the initial biopsy. From the case records and endoscopy reports it was clear that the carcinoma was present at the time of initial biopsy but had not been sampled at endoscopy; (iv) three patients died within one year of "metastatic adenocarcinoma, primary unknown". If any were gastric primaries it seems unlikely that they could have developed de novo and disseminated within such a short period before death; $(v)$ two patients seemed genuinely to develop gastric carcinoma subsequent to the 1976 biopsy. One had had a gastroenterostomy in 1934, type II intestinal metaplasia in the 1976 biopsy, and gastric adenocarcinoma diagnosed in 1979. The other patient had type III intestinal metaplasia in the 1976 biopsy taken after dilatation of a benign oesophageal stricture; he developed adenocarcinoma of the gastric fundus in 1981. Thus only two of the 174 study patients (one with type II intestinal metaplasia and one with type III) developed gastric carcinoma subsequent to the 1976 biopsy.

The 68 patients developing gastric carcinoma in 1985 whose case records were reviewed comprised 47 men and 21 women (mean (SD) age $71 \cdot 0(11 \cdot 4)$ years). After exclusion of non-malignant biopsy specimens within the three months prior to the diagnosis of gastric carcinoma (which on investigation were all false negative results due to endoscopic sampling error) only two patients were found to have had a previous gastric biopsy. One of these, a 1980 biopsy showed type II intestinal metaplasia; the other, a 1968 biopsy of the gastro-oesophageal junction, showed no intestinal metaplasia.

Table Intestinal metaplasia subtypes, patient data, and subsequent gastric carcinomas

\begin{tabular}{|c|c|c|c|c|c|}
\hline & \multirow[b]{2}{*}{ No of patients } & \multicolumn{2}{|c|}{ Sex } & \multirow[b]{2}{*}{ Mean (SD) age } & \multirow{2}{*}{$\begin{array}{l}\text { Subsequent } \\
\text { gastric carcinomas }\end{array}$} \\
\hline & & $M$ & $\boldsymbol{F}$ & & \\
\hline $\begin{array}{l}\text { No intestinal metaplasia } \\
\text { Type I } \\
\text { Type II } \\
\text { Type III }\end{array}$ & $\begin{array}{l}130 \\
16 \\
14 \\
14\end{array}$ & $\begin{array}{r}64 \\
8 \\
8 \\
12\end{array}$ & $\begin{array}{r}66 \\
8 \\
6 \\
2\end{array}$ & $\begin{array}{l}59 \cdot 2(16 \cdot 1) \\
66 \cdot 9(11 \cdot 5) \\
64 \cdot 3(12 \cdot 9) \\
65 \cdot 4(11 \cdot 6)\end{array}$ & $\begin{array}{l}0 \\
0 \\
1 \\
1\end{array}$ \\
\hline
\end{tabular}




\section{Discussion}

Tayside region, which encompasses the Dundee and Angus districts, has an average annual age standardised registration rate for gastric carcinoma of 24.27/100000 men, the highest rate in Scotland. ${ }^{11} \mathrm{~A}$ screening test which identified patients at increased risk of gastric carcinoma would therefore be expected to have a high pick up rate when applied to this area, but only one of 14 patients in our series with type III intestinal metaplasia developed gastric carcinoma over a 10 to 11 year period. Although numbers are small, this suggests that detection of type III intestinal metaplasia by biopsy is of low specificity as a predictor of risk of gastric carcinoma-at least over a decade. This confirms the suggestion of Murray et al $^{\mathbf{1 2}}$ that the prevalence of intestinal metaplasia containing sulphocumin in benign conditions would limit its routine use for identification of patients requiring follow up. Our findings are also in accord with those of Ectors and Dixon, ${ }^{13}$ who found that only one patient of 90 with intestinal metaplasia positive for sulphomucin developed gastric carcinoma when followed up for eight to nine years. They concluded that intestinal metaplasia positive for sulphomucin did not identify a high risk group and was of no value in surveillance for gastric carcinoma. Three methodological differences between that study and ours are worthy of note:

(1) We used the classification of intestinal metaplasia of Filipe $e t$ al $^{6}$ which is widely accepted and, we found, easy to apply.

(2) We screened biopsy specimens for intestinal metaplasia using haematoxylin and eosin stained sections, applying mucin histochemistry only to those specimens showing intestinal metaplasia. We believe that this approach closely simulates routine practice. (3) We included material from patients with partial gastrectomies as these patients remain at risk of gastric carcinoma.

Our findings and those of Ectors et al $^{13}$ seem to be at variance with studies ${ }^{69}$ showing type III intestinal metaplasia to be significantly associated with gastric carcinoma compared with benign gastric disease. The possibility has been raised that intestinal metaplasia positive for sulphomucin may be an epiphenomenon with respect to gastric carcinoma, both lesions tending to occur in the stomachs of older patients. ${ }^{13}$ In our study the group with type III intestinal metaplasia were significantly older than those without intestinal metaplasia. The groups with types I and II intestinal metaplasia were also older than the group without. These findings are similar to those of Rothery et $\mathrm{l}^{14}$ and mitigate against a simple age effect selectively producing an association between gastric carcinoma and type III intestinal metaplasia.
We believe that our data showing only a low proportion of patients with type III intestinal metaplasia developing gastric carcinoma are not necessarily incompatible with the findings of previous studies showing type III intestinal metaplasia to be significantly more common in gastric carcinoma than in benign gastric disease. The probable reason is apparent if we look at the likely outcome for patients presenting for endoscopy and biopsy who do not already have gastric carcinoma. Of these patients, only a small number will be destined to develop gastric carcinoma while a much larger number will never develop gastric carcinoma. Although a high proportion of the former very small group may show type III intestinal metaplasia, this will yield only a small number of patients with type III intestinal metaplasia. If even a small proportion of the others (who will never develop gastric carcinoma) show type III intestinal metaplasia this will produce a sufficiently large number of such patients to swamp the small numbers of patients from the former group. The specificity of the screening system will therefore be reduced.

In a study of this type the possibility must be considered that we have not followed up the patients with type III intestinal metaplasia long enough to allow gastric carcinoma to develop. This is unlikely because both the 1976 patients with type III intestinal metaplasia (mean age 65.4 years) and the 1985 patients with gastric carcinoma (mean age 71.0 years) were drawn from the same population base. If type III intestinal metaplasia generally progressed to gastric carcinoma the progression time would on average therefore be expected to be of the order of 71.0-65.4 $=5.6$ years. Our follow up of 10 to 11 years was roughly double this and if type III intestinal metaplasia did usually progress to gastric carcinoma we would have expected a large proportion of the gastric carcinomas to have developed during the study period. The fact that only one of 14 patients developed gastric carcinoma suggests that this progression does not occur. Indeed, the natural history of intestinal metaplasia is poorly understood and the concept of development of gastric carcinoma from intestinal metaplasia has been challenged on epidemiological grounds. ${ }^{15}$

Further problems arise from the lack of sensitivity of endoscopic biopsy as a means of detecting type III intestinal metaplasia. One of the two patients developing gastric carcinoma in this study did not have type III intestinal metaplasia at the time of the 1976 biopsy. Our numbers are small of course, but type III intestinal metaplasia is a focal lesion and may be missed on endoscopic biopsy as a result of sampling error. ${ }^{9}$

Leaving aside questions of sensitivity and specificity, a more fundamental problem for any 
health screening method arises if the at risk population is not being screened. One might imagine that patients undergoing gastroscopy for benign conditions might form an at risk group as it has been shown that patients with gastritis have an increased incidence of gastric carcinoma. ${ }^{16}$ We found, however, that only two of 68 patients presenting with gastric carcinoma in 1985 had ever had upper gastrointestinal endoscopy and biopsy despite a high rate of endoscopy in this area and its availability for more than a decade. Thus even if type III intestinal metaplasia detection was a good predictor of gastric carcinoma risk, its impact on the incidence and morbidity in the community would be minimal unless there was a radical increase in endoscopy rate. Even this is doubtful as Holdstock et al ${ }^{17}$ have shown that a 10-fold increase in endoscopy uptake in one area did not increase the detection rate of early gastric cancer.

It can be argued that the question of predictive value of type III intestinal metaplasia for subsequent gastric carcinoma can be more precisely answered by prospective studies. We believe, however, that guidelines on management of these patients are urgently required and that a retrospective approach is justified. Many pathologists and clinicians will be considering routine mucin histochemistry on gastric biopsy specimens with regular endoscopic follow up of patients with type III intestinal metaplasia. Such a policy would require reallocation of scarce laboratory and clinical resources. On the basis of the available information we conclude that such an approach is not justified and that detection of type III intestinal metaplasia is of limited value as an indicator of risk of gastric carcinoma.

We gratefully acknowledge the skilled technical assistance of Ms E Glass.

\section{References}

1 Morson BC. Intestinal metaplasia of the gastric mucosa. Br J Cancer 1955;9:365-76.

2 Teglbjaerg PS, Nielsen HO. "Small Intestinal Type" and "Colonic Type" intestinal metaplasia of the human stomach, and their relationship to the histogenetic types of gastric adenocarcinoma. Acta Pathol Microbiol Scand A 1978;86: 351-5.

3 Sipponen P, Seppala K, Varis K, et al. Intestinal metaplasia with colonic-type sulphomucins in the gastric mucosa; its association with gastric carcinoma. Acta Pathol Microbiol Scand A 1980;88:217-24.

4 Segura DI, Montero D. Histochemical characterisation of different types of intestinal metaplasia in gastric mucosa. Cancer 1983;52:498-503.

5 Jass JR. Role of intestinal metaplasia in the histogenesis of gastric carcinoma. J Clin Pathol 1980;33:801-10.

6 Filipe MI, Potet F, Bogomoletz WV, et al. Incomplete sulphomucin-secreting intestinal metaplasia for gastric cancer. Preliminary data from a prospective study from three centres. Gut 1985;26:1319-26.

7 Matsukura N, Suzuki K, Kawachi T, et al. Distribution of marker enzymes and mucin in intestinal metaplasia in human stomach and relation of complete and incomplete types of intestinal metaplasia to minute gastric carcinoma. JNCI 1980;65:231-40.

8 Jass JR, Filipe MI. A variant of intestinal metaplasia associated with gastric carcinoma: a histochemical study. Histopathology 1979;3:191-9.

9 Silva S, Filipe MI. Intestinal metaplasia and its variants in the gastric mucosa of Portuguese subjects: a comparative analysis of biopsy and gastrectomy material. Hum Pathol 1986;17:988-95.

10 Bancroft JD, Stevens A. Theory and practice of histological techniques. 2nd ed. London: Churchill Livingstone, 1983:192-205.

11 Scottish Cancer Registration Scheme. Cancer registration statistics-Scotland 1971-1980. Edinburgh: Scottish Health Service Common Services Agency 1984:71.

12 Murray LA, Williams GT. Sulphomucin-containing intestinal metaplasia in gastric biopsies-one year's experience. $J$ Pathol 1984;142:24-5.

13 Ectors N, Dixon JF. The prognostic value of sulphomucin positive intestinal metaplasia in the development of gastric cancer. Histopathology 1986;10:1271-7.

14 Rothery GA, Day DW. Intestinal metaplasia in endoscopic biopsy specimens of gastric mucosa. J Clin Pathol 1985;38:613-21.

15 Imai T, Murayama H. Time trend in the prevalence of intestinal metaplasia in Japan. Cancer 1983;52:353-61.

16 Siurala M, Lehtola J, Ihamaki T. Atrophic gastritis and its sequelae. Results of 19-23 years follow-up examinations. Scand J Gastroenterol 1974;9:441-6.

17 Holdstock G, Bruce S. Endoscopy and gastric cancer. Gut 1981;22:673-6.

18 Registrar Gezeral, Scotland. Annual report. Edinburgh: HMSO, 1984:100.

Requests for reprints to: Dr KCRB Ramesar, Pathology Department, Ninewells Hospital and Medical School, PO Box 120, Dundee DD1 9SY, Scotland. 